\title{
Modern concepts in cardio-oncology
}

\section{Tienush Rassaf, Matthias Totzeck}

Department of Cardiology and Vascular Medicine, West German Heart and Vascular Center, Medical Faculty, University Hospital Essen, 45147 Essen, Germany

Contributions: (I) Conception and design: All authors; (II) Administrative support: T Rassaf; (III) Provision of study materials or patients: None; (IV) Collection and assembly of data: None; (V) Data analysis and interpretation: None; (VI) Manuscript writing: All authors; (VII) Final approval of manuscript: All authors.

Correspondence to: Prof. Tienush Rassaf. Director and Chair, Department of Cardiology and Vascular Medicine, West German Heart and Vascular Center, Medical Faculty, University Hospital Essen, Hufelandstr. 55, 45147 Essen, Germany. Email: Tienush.Rassaf@uk-essen.de.

\begin{abstract}
Cardio-oncology has emerged as best option for many patients with cardiovascular complications related to cancer and cancer therapy. Classical chemotherapy, targeted and immune therapies as wells as radiotherapy challenge the cardiovascular system at multiple levels, including increased rates of e.g., hypertension, coronary artery disease, cardiomyopathy, arrhythmia, and thrombosis. The cardiologist working within the cardio-oncology team is confronted with a broad spectrum of therapies and combination protocols. Evidence from the past few years implicate that at least 30 novel cancer drugs can be expected to receive approval each year. The rate and extent of cardiovascular complications particularly for these new therapies remains to be evaluated. Overall, cardio-oncology has several important tasks to establish an optimal care for cancer patients: (I) risk assessment before therapy to prevent onset of cardiovascular disease, (II) assessment of diagnostic pathways to provide a timely diagnosis of early stages of cardiovascular complications, (III) characterize the extent of morbidity and mortality as induced by cancer therapies, (IV) establish therapeutic options to treat cardiotoxicity and (V) monitor long-term cancer survivors. In this special edition, experts in the field provide an overview about key concepts in cardio-oncology. This is complemented by general considerations about novel concepts in cardio-oncology including the establishment of fellowship programs, ethical issue and cancer survivorship programs as outlined in the present review.
\end{abstract}

Keywords: Cardio-oncology; cancer survivorship; cardiotoxicity; cardiomyopathy

Submitted Nov 21, 2018. Accepted for publication Nov 22, 2018.

doi: $10.21037 /$ jtd.2018.11.110

View this article at: http://dx.doi.org/10.21037/jtd.2018.11.110

\section{Introduction}

Recent advancements for therapies have increased the short and long-term survival of cardiological and oncological patients significantly. Cancer survival may, however, come with a prize-increased rates of cardiovascular events. Recent developments suggest that cardio-oncology is a distinct subspecialty in cardiology. This refers to the broad spectrum of new and established therapies and the variety of cardiovascular adverse events (1-3). The upsurge in scientific evidence in the field of cardio-oncology could now lead to the establishment of unified diagnostic and therapeutic algorithms, which are currently unavailable for most cancer therapies. Apart from the above-mentioned aspects, optimal cardio-oncology care should consider several essential concepts. This includes the establishment of novel structures, educational fellowship programs, novel detection strategies, ethical considerations, and the assessment of long-term outcomes.

\section{Institutional recommendations}

Cardio-oncology requires inter-disciplinary collaboration on multiple levels (4). Rapid detection of cardiotoxicity 
requires access to state-of-the art cardiovascular imaging from strain echocardiography to magnetic resonance tomography imaging and nuclear cardiology (PET/CT, PET/MRI). Development of new imaging techniques for the detection of fibrosis, amyloidosis and tumor infiltration into the myocardium is one of the major research challenges to cardio-oncology. Furthermore, a close interaction with the treating oncologist must be established on a personal level (interaction for individual decision-making) and through participation in regular conferences that discuss treatment management for patients with cardiotoxicity. Finally, many novel therapies have unknown cardiovascular risk profiles. Systematic assessment of the incidence rates, relative risk and involvement in clinical trials that assess the efficacy of cardiotoxicity treatment in the scope of clinical trial unit are essential part of cardio-oncology research.

\section{Cardio-oncology fellowships}

Cardio-oncology units have been established at many cardiovascular centers. The basis is a considerable and necessary interaction between the oncology and the cardiology department. Both disciplines have developed highly specialized diagnostic and therapeutic tools over the past decades. The cardiologist needs to be informed about the general indications for specific tumor therapies, life expectancies and possible cardiovascular risks $(2,3)$. This can only be realized through intense interaction with the oncologist at the individual patient level as well as in conferences, which continuously discuss strategies for the management of patients. A cardio-oncology fellowship has been proposed by several cardiac societies. However, it has just been established at very few sites so far. The respective training should provide the skills required for in- and outpatient cardio-oncology care. Several authors argue that the trainee should have treated at least 100 patients over at least one year of training $(4,5)$. Focus should be on the most common cardiovascular events from highly toxic cancer therapies including classical chemotherapy (anthracycline, cyclophosphamide), targeted therapies (tyrosine kinase inhibitors), immune checkpoint inhibitors and the sequelae of radiation. Profound knowledge is required for the combined used of biomarkers and imaging including speckle tracking strain imaging for an early detection of cardiotoxicity as outlined in the special reviews in this edition of the Fournal of Thoracic Diseases. Furthermore, several cancer treatments may generate acute and severe cardiovascular disease including cardiogenic shock and acute myocardial infarction. Trainees are recommended to be taught in emergency acute cardiac care, intensive care medicine and the management of complex arrhythmia (4).

An additional area of focus is the diagnosis of cardiac tumors. Primary malignant cardiac tumors and metastasis are very rare. Treatment is often realized together with an expert oncologist. This field is also covered by cardio-oncology and should be included in the training process together with a participation in tumor and heart team conferences.

Finally, long-term cancer survivors-a continuously growing group of patients-may present with symptoms suggesting late cardio-toxicity decades after the completion of cancer therapy. These symptoms may be particularly difficult to interpret as they may differ from presentation seen in canonical heart disease (e.g., fatigue and palpitations). Generally, coronary artery disease may become clinically relevant ten years after radiation therapy, while significant valvular heart disease may develop over two decades (6-9). The fellowship program should therefore include the assessment of long-term cancer survivors to provide an early detection and management of heart disease. Apart from the complete spectrum of cardiovascular medicine, the cardiooncology subspecialty therefore requires more general internal medicine training and a close interaction with the oncology department.

\section{Ethical considerations}

Prevention of cardiotoxicity is one of the key goals of cardiooncology. This includes the timely diagnosis of early stages of cardiomyopathy. In anthracycline recipients, cardiotoxicity may be detected using global longitudinal strain from speckle tracking echocardiography imaging. Reduced strain values may become evident months before left ventricular ejection fraction is reduced to levels that implicate toxic cardiomyopathy (10). Clinical signs may be completely absent at this stage (11). This may, however, advance into clinically apparent heart failure. Questions remain regarding the consequent management of these patients. Withdrawal of cancer drugs can be associated with significantly impaired survival. Heart failure therapies using beta-blockers and angiotensin-converting enzyme inhibitors have been tested in several trials with inconclusive benefit (12-15). The cardio-oncology teams therefore needs to discuss with the oncologist the following issues: (I) follow-up intervals, (II) dose reduction/application form of chemotherapy, (III) temporary or permanent termination of therapy, (IV) alternative strategies, and (V) heart failure therapy. 
This does not only hold true for classical cancer therapy. BRAF/MEK inhibitors and immune checkpoint inhibitors are novel therapies that are of particular importance for the treatment of advanced stage melanoma (16). Using these options, average survival for melanoma stage IV can be prolonged by several months in these patients (17-20). BRAF/MEK and other small molecule inhibitor therapy may induce heart failure, while immune checkpoint inhibitor therapy has been associated with myocarditis (21-23). Particularly the latter is difficult to diagnose and may require the combined use of biomarkers, nuclear imaging (PET) and endomyocardial biopsy (24). In case of severe forms of myocarditis or heart failure, administration of both cancer drug entities should be terminated until cardiovascular disease has been managed. However, withdrawal at every marginal sign of cardiotoxicity may exempt many patients from therapy and thus reduce life expectancy. The decision for the temporary or permanent cessation of cancer drugs can therefore only by made in cardio-oncology teams together with the oncologists.

Finally, many society recommendations argue in favor of a strict management of cardiovascular risk factors during and after cancer therapy. This relates e.g., to the treatment of hypertension, dyslipidemia and diabetes mellitus (1,25-27). Optimal handling of these cardiovascular risk factors has been associated with a better prognosis in cancer patients. However, the extensive use of risk factor modifying drugs in advance cancer patients e.g., in head and neck cancer with poor prognosis again requires an individual decisionmaking. Other treatment modalities (nutrition, pain management) may play a much more important role.

\section{Cancer survivorship}

The majority of consultation in cardio-oncology is related to patients with complications resulting from an ongoing cancer therapy. However, increasing numbers of survivors of childhood malignancies (particularly leukemia and lymphoma requiring myeloablative chemotherapy in combination with radiation) report to the corresponding cardio-oncology service. At considerable young age, these patients often present with premature cardiovascular disease and yet unusual symptoms. The risk of cardiovascular disease causing significant morbidity and mortality in $30-40$ years old patients may be comparable to non-cancer cohort at the age of 60-70 (28). Yet the majority will experience treatment-related consequences at some point in their lives. Significant impairment of left ventricular functions, coronary artery disease and valvular heart disease are the main cardiovascular complications following decades after cancer therapy completion. A systematic approach for these patients is missing. Diagnosis of cancer and the precise treatments may have been lost over the years so that the complete risks for heart disease can often only be estimated. The development of cardiovascular risk factors, such as hypertension, diabetes, and dyslipidaemia, should also be closely monitored (29). Exposure to radiation provokes cardiovascular disease years or even decades after therapy. Patients with symptoms should be referred to cardiologists for evaluation including imaging and, if indicated, coronary angiography. Systematic assessment of heart disease should be considered 5-10 years after completion of therapy. This includes cardiovascular imaging and ergometric testing. Cardiovascular risk factor assessment and treatment is recommended according to current guidelines.

\section{Detection of cardiotoxicity-novel approaches in cardio-oncology}

Many previous strategies have focused on an early detection of cardiotoxicity and a specific treatment hereafter. Conventional cardiac biomarkers and echocardiography have been discussed and a widely applied in cardio-oncology practice. Many novel therapies have yet been incompletely defined regarding their effects for the cardiovascular system. Small molecule tyrosine kinase inhibitors target aberrant kinase signaling in a wide variety of malignancy. A large group of therapies targets the vascular endothelial growth factor (VEGF) pathway. Comparable kinases are expressed in the cardiovascular system (30). In some patients, VEGF inhibitor administration may lead to hypertension, arterial and venous thrombosis, ischemia, stroke and an increase risk of bleeding (22). It is unclear why some patients are particularly susceptible to VEGF complications while the majority will not have any adverse events. Preclinical studies have tried to recapitulate the kinase system in animal models but the human and rodent kinase pathways show broad differences. Novel approaches try to assess kinase inhibitors and cellular dysfunction in cells and tissues of individual patients. This could provide a pre-treatment diagnostic tool to prevent cardiotoxicity patients at risk as the first specific cardio-oncology diagnostic test (31).

\section{Conclusions}

It has been widely accepted for many decades that specific 
cancer therapies may induce a profound damage to the cardiovascular system. State-of-the art cancer therapy now involves a wide variety of classical, targeted and immune therapies in combination with radiation. First, second, third and fourth line treatment options are available for many cancers. This has turned many cancers into chronic diseases. Cancer survivors suffer from cardiovascular disease, which limits the quality of life and prognosis. Cardio-oncology has the goals to quantify the extent of cardiovascular adverse events, detect cardiotoxicity at early stages and provide treatment algorithms

As outlined in this review, particular focus must be on individual decision-making. Withdrawal of potentially curative drugs to prevent cardiovascular disease progression must be balanced to the caveats of heart disease. Risk factor optimization (hypertension, dyslipidemia etc.) may be an optimal treatment goal in otherwise healthy breast cancer patients. In multimorbid heck and neck cancer patients, who might have difficulties to shallow, other goals may be more important (palliative care).

The information about cardio-oncology are constantly growing and can hardly be summarized in one review article. This special issue of the Fournal of Thoracic Disease therefore compiles individual articles of experts in the field addressing the key issues of imaging, biomarkers, breast cancer, heart failure and the treatment options as realized in cardio-oncology teams.

\section{Acknowledgements}

None.

\section{Footnote}

Conflicts of Interest: The authors have no conflicts of interest to declare.

\section{References}

1. Zamorano JL, Lancellotti P, Rodriguez Munoz D, et al. 2016 ESC Position Paper on cancer treatments and cardiovascular toxicity developed under the auspices of the ESC Committee for Practice Guidelines: The Task Force for cancer treatments and cardiovascular toxicity of the European Society of Cardiology (ESC). Eur Heart J 2016;37:2768-801.

2. Chang HM, Moudgil R, Scarabelli T, et al. Cardiovascular Complications of Cancer Therapy: Best Practices in
Diagnosis, Prevention, and Management: Part 1. J Am Coll Cardiol 2017;70:2536-51.

3. Chang HM, Okwuosa TM, Scarabelli T, et al. Cardiovascular Complications of Cancer Therapy: Best Practices in Diagnosis, Prevention, and Management: Part 2. J Am Coll Cardiol 2017;70:2552-65.

4. Johnson MN, Steingart R, Carver J. How to Develop a Cardio-oncology Fellowship. Heart Fail Clin 2017;13:361-6.

5. Lenihan DJ, Hartlage G, DeCara J, et al. CardioOncology Training: A Proposal From the International Cardioncology Society and Canadian Cardiac Oncology Network for a New Multidisciplinary Specialty. J Card Fail 2016;22:465-71.

6. Cheng YJ, Nie XY, Ji CC, et al. Long-Term Cardiovascular Risk After Radiotherapy in Women With Breast Cancer. J Am Heart Assoc 2017;6.

7. Cuomo JR, Sharma GK, Conger PD, et al. Novel concepts in radiation-induced cardiovascular disease. World J Cardiol 2016;8:504-19.

8. Cutter DJ, Schaapveld M, Darby SC, et al. Risk of valvular heart disease after treatment for Hodgkin lymphoma. J Natl Cancer Inst 2015;107.

9. Darby SC, Ewertz M, Hall P. Ischemic heart disease after breast cancer radiotherapy. N Engl J Med 2013;368:2527.

10. Cardinale D, Colombo A, Lamantia G, et al. Anthracycline-induced cardiomyopathy: clinical relevance and response to pharmacologic therapy. J Am Coll Cardiol 2010;55:213-20.

11. Thavendiranathan P, Poulin F, Lim KD, et al. Use of myocardial strain imaging by echocardiography for the early detection of cardiotoxicity in patients during and after cancer chemotherapy: a systematic review. J Am Coll Cardiol 2014;63:2751-68.

12. Bosch X, Rovira M, Sitges M, et al. Enalapril and carvedilol for preventing chemotherapy-induced left ventricular systolic dysfunction in patients with malignant hemopathies: the OVERCOME trial (preventiOn of left Ventricular dysfunction with Enalapril and caRvedilol in patients submitted to intensive ChemOtherapy for the treatment of Malignant hEmopathies). J Am Coll Cardiol 2013;61:2355-62.

13. Avila MS, Ayub-Ferreira SM, de Barros Wanderley Junior MR, et al. Carvedilol for Prevention of Chemotherapy Related Cardiotoxicity. J Am Coll Cardiol 2018.

14. Gulati G, Heck SL, Ree AH, et al. Prevention of cardiac dysfunction during adjuvant breast cancer therapy (PRADA): a 2 × 2 factorial, randomized, placebocontrolled, double-blind clinical trial of candesartan and 
metoprolol. Eur Heart J 2016;37:1671-80.

15. Pituskin E, Mackey JR, Koshman S, et al. Multidisciplinary Approach to Novel Therapies in Cardio-Oncology Research (MANTICORE 101-Breast): A Randomized Trial for the Prevention of Trastuzumab-Associated Cardiotoxicity. J Clin Oncol 2017;35:870-7.

16. Schadendorf D, van Akkooi ACJ, Berking C, et al. Melanoma. Lancet 2018;392:971-84.

17. Long GV, Hauschild A, Santinami M, et al. Adjuvant Dabrafenib plus Trametinib in Stage III BRAF-Mutated Melanoma. N Engl J Med 2017;377:1813-23.

18. Larkin J, Chiarion-Sileni V, Gonzalez R, et al. Combined Nivolumab and Ipilimumab or Monotherapy in Untreated Melanoma. N Engl J Med 2015;373:23-34.

19. Robert C, Long GV, Brady B, et al. Nivolumab in previously untreated melanoma without BRAF mutation. N Engl J Med 2015;372:320-30.

20. Long GV, Stroyakovskiy D, Gogas H, et al. Combined BRAF and MEK inhibition versus BRAF inhibition alone in melanoma. N Engl J Med 2014;371:1877-88.

21. Mahmood SS, Fradley MG, Cohen JV, et al. Myocarditis in Patients Treated With Immune Checkpoint Inhibitors. J Am Coll Cardiol 2018;71:1755-64.

22. Totzeck M, Mincu RI, Mrotzek S, et al. Cardiovascular diseases in patients receiving small molecules with antivascular endothelial growth factor activity: A meta-analysis of approximately 29,000 cancer patients. Eur J Prev Cardiol 2018;25:482-94.

23. Livingstone E, Zimmer L, Vaubel J, et al. BRAF, MEK and KIT inhibitors for melanoma: adverse events and their management. Chin Clin Oncol 2014;3:29.

Cite this article as: Rassaf T, Totzeck M. Modern concepts in cardio-oncology. J Thorac Dis 2018;10(Suppl 35):S4386-S4390. doi: $10.21037 /$ jtd.2018.11.110
24. Brahmer JR, Lacchetti C, Schneider BJ, et al. Management of Immune-Related Adverse Events in Patients Treated With Immune Checkpoint Inhibitor Therapy: American Society of Clinical Oncology Clinical Practice Guideline. J Clin Oncol 2018;36:1714-68.

25. Mehta LS, Watson KE, Barac A, et al. Cardiovascular Disease and Breast Cancer: Where These Entities Intersect: A Scientific Statement From the American Heart Association. Circulation 2018;137:e30-66.

26. Virani SA, Dent S, Brezden-Masley C, et al. Canadian Cardiovascular Society Guidelines for Evaluation and Management of Cardiovascular Complications of Cancer Therapy. Can J Cardiol 2016;32:831-41.

27. Williams B, Mancia G, Spiering W, et al. 2018 ESC/ESH Guidelines for the management of arterial hypertension. Eur Heart J 2018;39:3021-104.

28. Armstrong GT, Kawashima T, Leisenring W, et al. Aging and risk of severe, disabling, life-threatening, and fatal events in the childhood cancer survivor study. J Clin Oncol 2014;32:1218-27.

29. Armenian SH, Lacchetti C, Barac A, et al. Prevention and Monitoring of Cardiac Dysfunction in Survivors of Adult Cancers: American Society of Clinical Oncology Clinical Practice Guideline. J Clin Oncol 2017;35:893-911.

30. Moslehi JJ. Cardiovascular Toxic Effects of Targeted Cancer Therapies. N Engl J Med 2016;375:1457-67.

31. Sharma A, Burridge PW, McKeithan WL, et al. Highthroughput screening of tyrosine kinase inhibitor cardiotoxicity with human induced pluripotent stem cells. Sci Transl Med 2017;9. 